\title{
FREE AXIAL VIBRATION OF A NANOROD USING THE WKB METHOD
}

\author{
Anita Ciekot \\ Institute of Mathematics, Czestochowa University of Technology Czestochowa, Poland \\ anita.ciekot@im.pcz.pl
}

\begin{abstract}
In this paper the solution of the vibration problem of a non-uniform nanorod is presented. The WKB method is applied to solve the equation of motion for free axial vibration of the nanorod.
\end{abstract}

\section{Introduction}

The vibration analysis of nanostructures has been of great interest because of their applications, for example in nanoelectromechanical, nanodevices. The scale effect on vibration characteristics of the nanostructures (nanotubes, nanobeams, nanorods) is often investigated by using the non-local elasticity theory.

The axial vibrations of nanorods are induced by the axial external forces. The frequencies of the axial free vibration of a nanorod are important parameters which characterize the behaviour of this nanorod during the enforced vibration. The axial free vibrations of non-uniform nanorods were studied in papers [1-4].

The small scale effect on vibration of non-uniform nanorods by using the theory of non-local elasticity was investigated by Chang [1]. The numerical solutions was obtained by using the differential quadrature method. The effect of the nonlocal long-range interactions on the longitudinal vibration of a nanorod was the subject of the paper [2] by Huang. The exact solution of the problem of free and forced vibration was determined under the condition of a uniform non-local kernel. The axial vibration of a tapered nanorod was studied by Mohammad Danesh et al. [3]. The solution of the governing equations of the nanorod vibration was obtained by using the differential quadrature method. The same problem was studied by Lee, using finite element method. Firouz-Abadi investigated the three dimensional free vibration properties of nanocones based on a nonlocal continuum shell model and the Galerkin technique [4]. A modified Wentzel-Kramers-Brillouin (WKB) method to obtain an asymptotic solution of vibration problem of nanocones was applied by Guo and Yang [5].

The free vibration of a non-uniform nanorod is the subject of the present paper. The solution of the problem was derived by using the WKB method $[6,7]$. 


\section{Formulation of the problem}

The equation of motion for free-vibrating axial non-uniform nanorod in the nonlocal elasticity can be written in the following form:

$$
\frac{\partial}{\partial x}\left(E A(x) \frac{\partial u}{\partial x}\right)=\left(1-\left(e_{0} a\right)^{2} \frac{\partial^{2}}{\partial x^{2}}\right) m(x) \frac{\partial^{2} u}{\partial t^{2}}
$$

where: $u(x, t)$ - the axial displacement, $m(x)$ - the mass per unit length, $E(x)$ - the Young's modulus, $A(x)$ is the area of cross-section of the non-uniform and non-homogeneous nanorod, $e_{0}$ is a constant appropriate to each material and $a$ is an internal characteristic size. The function $u(x, t)$ satisfies the boundary conditions

$$
u(0, t)=u(L, t)=0
$$

\section{Solution of the problem}

In order to find the natural frequencies of the nanorod, one assumes a solution of the problem in the form:

$$
u(x, t)=U(x) e^{i \omega t}
$$

Substituting (3) into Eq. (1) gives:

$$
\frac{d}{d x}\left(E A(x) \frac{d U}{d x}\right)+\omega^{2}\left(1-\left(e_{0} a\right)^{2} \frac{d^{2}}{d x^{2}}\right) m(x) U(x)=0
$$

Introducing a new variable $\quad \xi=\frac{x}{L}$ and assuming $m(\xi)=\rho A(\xi), A_{0}=A(0)$, $\rho, E$ - const in Eq. (4), one obtains:

$$
\frac{1}{L^{2}} \frac{d}{d \xi}\left(A(\xi) \frac{d U}{d \xi}\right)+\frac{\rho A_{0} \omega^{2} L^{2}}{E A_{0}}\left(1-\left(\frac{e_{0} a}{L}\right)^{2} \frac{d^{2}}{d \xi^{2}}\right) A(\xi) U(\xi)=0
$$

After transformation, Eq. (5) can be written in the form:

$$
p(\xi) \frac{d^{2} U}{d \xi^{2}}+q(\xi) \frac{d U}{d \xi}+r(\xi) U(\xi)=0
$$


where:

$$
\begin{gathered}
p(\xi)=\left[1-\Omega^{2}\left(\frac{e_{0} a}{L}\right)^{2}\right] A(\xi), \quad q(\xi)=\left[1-2 \Omega^{2}\left(\frac{e_{0} a}{L}\right)^{2}\right] A^{\prime}(\xi) \\
r(\xi)=\Omega^{2}\left[A(\xi)-\left(\frac{e_{0} a}{L}\right)^{2} A^{\prime \prime}(\xi)\right], \quad \Omega^{2}=\frac{\rho A_{0} \omega^{2} L^{2}}{E A_{0}}
\end{gathered}
$$

In order to use the WKB approximation, the first step is to transfer Eq. (6) by making the transformation

$$
U(\xi)=\Phi(\xi) V(s(\xi))
$$

where $\Phi(\xi), s(\xi), \quad V(s(\xi))$ are arbitrary functions, the Eq. (5) becomes

$$
\begin{aligned}
& p(\xi) \Phi(\xi)\left[s^{\prime}(\xi)\right]^{2} V^{\prime \prime}(s(\xi))+ \\
& +\left\{\left[2 p(\xi) \Phi^{\prime}(\xi)+q(\xi) \Phi(\xi)\right] s^{\prime}(\xi)+p(\xi) \Phi(\xi) s^{\prime \prime}(\xi)\right\} V^{\prime}(s(\xi)) \\
& +\left[p(\xi) \Phi^{\prime \prime}(\xi)+q(\xi) \Phi^{\prime}(\xi)+r(\xi) \Phi(\xi)\right] V(s(\xi))=0
\end{aligned}
$$

Equating the coefficient of $V^{\prime}(s(\xi))$ to zero gives an equation for $\Phi(\xi)$ :

$$
2 p(\xi) \Phi^{\prime}(\xi) s^{\prime}(\xi)+p(\xi) \Phi(\xi) s^{\prime \prime}(\xi)+q(\xi) \Phi(\xi) s^{\prime}(\xi)=0
$$

The solution of this equation is:

$$
\Phi(\xi)=\exp \left(-\int Q(\xi) d \xi\right)=A^{\mu}(\xi)
$$

where

$$
Q(\xi)=\frac{p(\xi) s^{\prime \prime}(\xi)+q(\xi) s^{\prime}(\xi)}{2 p(\xi) s^{\prime}(\xi)}, \quad \mu=\frac{1}{2\left(1-\Omega^{2}\left(\frac{e_{0} a}{L}\right)^{2}\right)}-1
$$

Taken into account Eq. (9) in (8), one obtains

$$
V^{\prime \prime}(s(\xi))+\frac{1}{\left[s^{\prime}(\xi)\right]^{2}}\left[Q^{2}(\xi)-Q^{\prime}(\xi)-\frac{q(\xi)}{p(\xi)} Q(\xi)+\frac{r(\xi)}{p(\xi)}\right] V(s(\xi))=0
$$

Let $s(\xi)=\frac{1}{v} \xi, \quad s^{\prime}(\xi)=\frac{1}{v}$, then the Eq. (11) can be evaluated as: 


$$
V^{\prime \prime}(s)+v^{2} F(s) V(s)=0
$$

where

$$
F(s)=\frac{A^{\prime}(\xi) q(\xi)+2 p(\xi)\left(2 \Omega^{2} A(\xi)-A^{\prime \prime}(\xi)\right)}{4 p^{2}(\xi)}, \quad \xi=v s
$$

and $v$ - is a large, positive number.

If $F(s)$ is constant the nature of the solution depends on its sign; for positive values, $F(s)=1$, the two linearly independent solutions are the oscillatory functions $V(s)=\exp ( \pm i v s)$; for negative values, $F(s)=-1$, the solutions are $V(s)=\exp ( \pm v s)$ which change exponentially. These limiting cases suggest expressing the solution of Eq. (12) in the form:

$$
V(s)=\left\{\begin{array}{lll}
\exp (i v \Psi(s)) & \text { if } \quad F(s)>0 \\
\exp (v \Psi(s)) & \text { if } \quad F(s)<0
\end{array}\right.
$$

for some function $\Psi(s)$. Substituting into equation (12) gives the following nonlinear equations for $\Psi(s)$ :

$$
\begin{aligned}
& \frac{i}{v} \Psi^{\prime \prime}(s)+F(s)-\left[\Psi^{\prime}(s)\right]^{2}=0 \quad \text { if } \quad F(s)>0 \\
& \frac{1}{v} \Psi^{\prime \prime}(s)+F(s)+\left[\Psi^{\prime}(s)\right]^{2}=0 \quad \text { if } \quad F(s)<0
\end{aligned}
$$

Since $v \gg 1$ an approximate solution is obtained by ignoring the first term, so the simplest approximate solutions of equation (14) are

$$
V(s)= \begin{cases}\exp \left( \pm i v \int \sqrt{F(s)} d s\right) & \text { if } \quad F(s)>0 \\ \exp \left( \pm v \int \sqrt{-F(s)} d s\right) & \text { if } \quad F(s)<0\end{cases}
$$

Using the WKB method solution of equation (12) may be obtained using the series expansion

$$
\Psi(s)=\Psi_{0}(s)+\frac{1}{v} \Psi_{1}(s)+\frac{1}{v^{2}} \Psi_{2}(s)+\ldots=\sum_{k=0}^{\infty} v^{-k} \Psi_{k}(s)
$$

Substituting this into (10) and equating the coefficient of $v^{-n}$ to zero gives an equation for $\Psi_{n}(s)$ in terms of $\Psi_{k}(s), \quad k=1,2, \ldots . n-1$. If $F(s)>0$ we obtain 


$$
\begin{array}{ll}
k=0: & \Psi_{0}^{\prime}(s)= \pm \sqrt{F(s)} \\
k=1: & i \Psi_{0}^{\prime \prime}(s)=2 \Psi_{0}^{\prime}(s) \Psi_{1}^{\prime}(s) \\
k=2: & i \Psi_{1}^{\prime \prime}(s)=2 \Psi_{0}^{\prime}(s) \Psi_{2}^{\prime}(s)+\left[\Psi_{1}^{\prime}(s)\right]^{2} \\
k=3: & i \Psi_{2}^{\prime \prime}(s)=2 \Psi_{0}^{\prime}(s) \Psi_{3}^{\prime}(s)+2 \Psi_{1}^{\prime}(s) \Psi_{2}^{\prime}(s)
\end{array}
$$

generally:

$$
i \Psi_{k-1}^{\prime \prime}(s)-\sum_{j=0}^{k} \Psi_{j}^{\prime}(s) \Psi_{k-j}^{\prime}(s)
$$

The solutions of the first and second of these equations are

$$
\Psi_{0}(s)= \pm \int \sqrt{F(s)} d s, \quad \Psi_{1}(s)=\frac{i}{4} \ln F(s)
$$

and hence

$$
V(s)=\frac{1}{(F(s))^{\frac{1}{4}}} \exp \left( \pm i v \int \sqrt{F(s)} d s\right)
$$

The general solution of equation (12) is then as follow:

for $F(s)>0$,

$$
V(s)=\frac{1}{(F(s))^{\frac{1}{4}}}\left\{A \exp \left(i v \int \sqrt{F(s)} d s\right)+B \exp \left(-i v \int \sqrt{F(s)} d s\right)\right\}
$$

and for $F(s)<0$,

$$
V(s)=\frac{1}{(F(s))^{\frac{1}{4}}}\left\{C \exp \left(v \int \sqrt{-F(s)} d s\right)+D \exp \left(-v \int \sqrt{-F(s)} d s\right)\right\}
$$

for some constants $A, B, C$ and $D$.

Inserting the obtained solution (Eq. (18) and (19)) into the boundary conditions $V(0)=0$ and $\quad V(1)=0$ yields the following set of homogeneous equations (for $F(s)>0)$ 


$$
\left\{\begin{array}{l}
\frac{1}{(F(0))^{\frac{1}{4}}}\{A \exp (i v f(0))+B \exp (-i v f(0))\}=0 \\
\frac{1}{(F(1))^{\frac{1}{4}}}\{C \exp (i v f(1))+D \exp (-i v f(1))\}=0
\end{array}\right.
$$

where $f(s)=\int \sqrt{F(s)} d s$.

The non-trivial solutions of this set of equations exist if and only if the determinant of the coefficient matrix is zero. This determinant defined a frequency function as follows:

$$
\left|\begin{array}{ll}
\exp (i v f(0)) & \exp (-i v f(0)) \\
\exp (i \vee f(1)) & \exp (-i v f(1))
\end{array}\right|=0
$$

The roots of equation (21) are called natural frequencies and can be determined numerically.

\section{Conclusions}

Then equation of motion for free-axial vibration of uniform nanorod was solved with the WKB approximate method. A boundary conditions were applied to obtain the natural frequencies which are relevant parameters for the behaviour of the tested nanorod. These solutions can be used in numerical frequency analysis of the non-uniform nanorods.

\section{References}

[1] Chang T.-P., Small scale effect on axial vibration of non-uniform and non-homogeneous nanorods, Computational Materials Science 2012, 54, 23-27.

[2] Huang Z., Nonlocal effects of longitudinal vibration in nanorod with internal long-range interactions, International J. of Solid and Structure 2012, 49, 2150-2154.

[3] Danesh M., Farajpour A., Mohammadi M., Axial vibration analysis of tapered nanorod based on nonlocal elasticity theory and differential quadrature method, Mechanics Research Communications 2012, 39, 23-27.

[4] Lee J.H., Lee B.S., Modal analysis of carbon nonotubes and nonocones using FEM, Comp. Material Science 2012, 51, 30-42.

[5] Shu-Qi Guo, Shao-Pu Yang, Axial vibration analysis of nanocones elasticity theory, Acta Mech. Sin. 2012, 28(3), 801-807.

[6] Nayfen A.H., Introduction to Perturbation Techniques, John Wiley \& Sons, Inc., 1981.

[7] Richards D., Advanced Mathematical Methods with Maple, Cambridge University Press, 2009. 